\title{
Self-assembled Nanostructures inside Indirect Bandgap Semiconductor by Using IR Femtosecond Double-pulses
}

\author{
T. Sei ${ }^{1}$, Y. Shimotsuma*1, M. Sakakura ${ }^{2}$ and K. Miura ${ }^{1}$ \\ ${ }^{* 1}$ Department of Material Chemistry Graduate School of Engineering, Kyoto University, Kyoto 615 - \\ 8510, Japan \\ E-mail: yshimo@func.mc.kyoto-u.ac.jp \\ *2 Society-Academia Collaboration for Innovation, Kyoto University, Kyoto 615-8510, Japan
}

\begin{abstract}
Periodic nanostructures inside gallium phosphide are successfully induced by the IR femtosecond laser pulses. At present, in the case of a single component glass and an indirect bandgap semiconductor, we empirically found that such self-assembled nanostructures can be formed. Based on the previous researches, we assumed that the analogy of the longer relaxation time of a photoexcited electron between a glass and an indirect bandgap semiconductor is related to the nanostructure formation. In order to elucidate the formation mechanism of the periodic nanostructures inside semiconductor materials, we have compared the photoinduced structures in a gallium arsenide and a gallium phosphide. We have also observed that the periodic nanostructure embedded in gallium phosphide exhibits high electrical conductivities.
\end{abstract}

DOI: 10.2961/jlmn.2016.01.0014

Keywords: indirect band gap, infrared femtosecond laser, nanostructures, self-assembly, gallium arsenide, gallium phosphide

\section{Introduction}

Various studies on the transformation from an insulator to a metal have been performed by the interaction between a solid material and an ultrashort pulse laser. For example, studies on formation of optical waveguide inside glass were performed because ultrashort pulse laser makes it possible to modify only the focal point [1]. And studies on realization of solar cell with higher energy conversion efficiencies were also performed by nanostructuring on the surface of silicon wafer like moth-eye structure [2]. In addition, many studies of micromachining on the surface of semiconductors also have been performed. It is well known that a laser induced periodic surface structures (LIPSS) can be formed on the surface of various solid materials [3]. Such LIPSS can be also induced on the surface of silicon [4] and compound semiconductors including indium phosphide (InP) [5] and gallium phosphide (GaP) [6] after irradiation with ultrashort laser pulses. At present, the formation mechanisms of LIPSS are advocated [7, 8], and have been still discussed. On the other hand, very few researches relating the photo-induced structure embedded in a semiconductor material by ultrashort pulse laser were reported. More recently, periodic nanostructure was successfully self-organized inside silicon single crystal (c-Si) by using the infrared femtosecond laser double-pulse configuration [9]. We have confirmed that such periodic nanostructures were aligned parallel to the polarization direction of the first arriving pulse. It should be noted that the nanogratings in fused silica are consistently aligned perpendicular to the polarization direction [10]. The periodic nanostructure composed of nano-scaled stripe-like strained silicon exhibiting high electron conductivity and low thermal conductivity. So we suggested that such nanostructure could act as a thermoelectric conversion material based on the phonon scattering without preventing electron motion.
Although such structural changes could be interpreted in terms of the light-plasma interactions enhanced by the femtosecond double-pulse trains, details of formation mechanism have not been fully understood. To reveal the mechanism of the nanostructuring in semiconductor, we compared the photoinduced structures in gallium arsenide (GaAs) and $\mathrm{GaP}$. Although these are the same crystal structure of zincblende, the bandgap structures are different, namely, GaAs (direct, $\mathrm{E}_{\mathrm{g}}=1.43 \mathrm{eV}$ ) and $\mathrm{GaP}$ (indirect, $\mathrm{E}_{\mathrm{g}}=2.26 \mathrm{eV}$ ).

\section{Experimental}

In the experiments we used commercially available (100) GaAs and GaP wafers with thicknesses of $500 \mu \mathrm{m}$. We typically used undoped $\mathrm{GaAs}$ and $\mathrm{GaP}$ wafers. In order to overcome the bandgap energy limit for GaAs, the experiments were performed using a mode-locked Cr: Forsterite femtosecond laser system equipped with a regenerative amplifier (Avesta; FREGAT-200), operating at $1.24 \mu \mathrm{m}$ with $110 \mathrm{fs}$ pulse duration and $1 \mathrm{kHz}$ repetition rate. To compensate a spherical aberration, the laser beam was focused inside wafer through the objective lens with a correction collar (Olympus; LCPLN-IR, 100, numerical aperture of 0.85). As a typical beam waist diameter and pulse energy were $5 \mu \mathrm{m}$ and $10 \mu \mathrm{J}$, respectively, the laser peak intensity was estimated to be $2 \times 10^{15} \mathrm{~W} / \mathrm{cm}^{2}$. The induced structures inside GaAs and GaP wafer were inspected by an optical microscope with infrared lamp (BHK; MAXIR) and InGaAs camera (Hamamatsu Photonics; C10633-13). Fig. 1 illustrates the femtosecond double-pulse configuration to induce structural change inside GaAs and GaP wafer. To promote the interaction between an excited electron and a photon [11], we employed the femtosecond double-pulse configuration. The linearly polarized double-pulse (the first arriving pulse: $E_{1}$, the second arriving pulse: $E_{2}$ ) was focused at the depth of 
$250 \mu \mathrm{m}$ from sample surface. The total pulse energy of the equally divided double-pulses with a delay time of 1 ps was typically set to be $10 \mu \mathrm{J}$ and $40 \mu \mathrm{J}$ for $\mathrm{GaP}$ and $\mathrm{GaAs}$ (i.e., each pulse energy was $5 \pm 0.5 \mu \mathrm{J}$ and $20 \pm 0.5 \mu \mathrm{J}$ ), respectively. In the characterization of the photoinduced structures, a confocal Raman spectrometer (Tokyo Instruments; NanoFinder 30) was used for structural identification. We have also analyzed the embedded structure in the following two methods: one is the polished sample surface to depth of the focal spot location, another is the cleaved and unpolished surface in the direction perpendicular to the laser traces. These surfaces were inspected by a scanning electron microscope (JEOL; JSM-6705F) and an Auger electron microscopy (Ulvac PHI; PHI-700). To evaluate the electrical conductivities after the laser irradiation, we have also performed the measurements of terahertz time domain spectroscopy (THz-TDS).

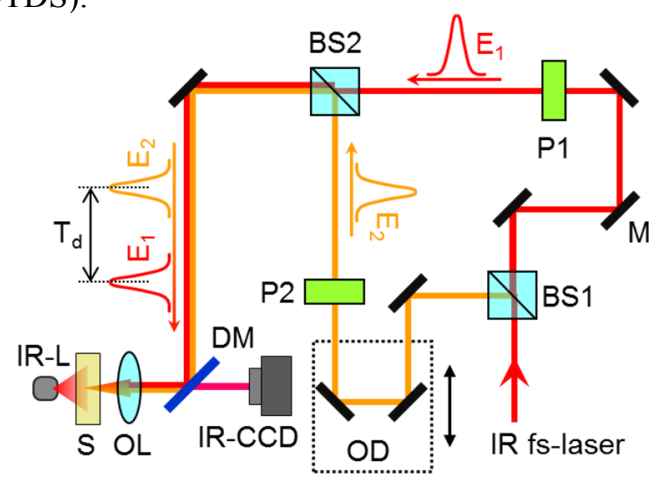

Fig. 1 Experimental setup of IR femtosecond double-pulse configuration: polarizing beam splitters (BS1, BS2), half-wave plate (P1, P2), optical delay line (OD), dichroic mirror (DM), microscope objective lens (OL). The sample (S) was placed on the XYZ stage of the transmitted light microscope equipped with the IR lamp (IR-L) and the InGaAs camera (IR-CCD).

\section{Results and discussion}

\subsection{Photoinduced structural change in GaAs}

Previously, we have confirmed that it is impossible to induce any structural changes in Si by the femtosecond single-pulse-train even if the pulse energy is $600 \mu \mathrm{J}$ [12]. We have also confirmed that the pulse energy of at least $20 \mu \mathrm{J}$ is required to induce the structural change without surface damage in GaAs by the single-pulse-train. Such situation that photoinduced structural change in GaAs is difficult is analogous to the case of Si. On the other hand, it is possible to induce structural change inside $\mathrm{GaP}$ by the single-pulsetrain with the pulse energy of more than $0.1 \mu \mathrm{J}$.

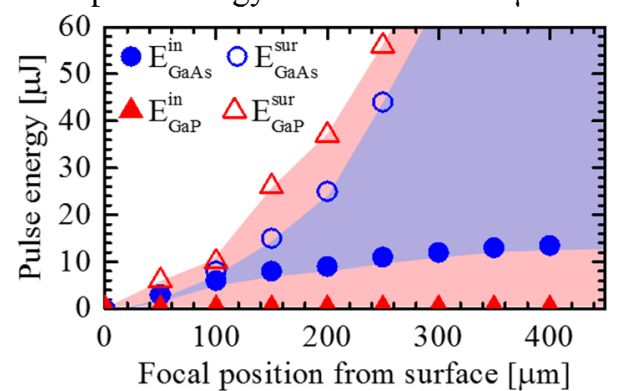

Fig. 2 Comparison of laser energy threshold for the internal structural change $\left(E^{\mathrm{in}}\right)$ and the surface ablation $\left(\mathrm{E}^{\mathrm{sur}}\right)$ as a function of the depth of focus from the sample surface. The blue and red areas show the conditions in which structural modification inside GaAs and $\mathrm{GaP}$, respectively, can be performed.
Previously we have reported that the shape of the photoinduced structures were elongated along with the polarization direction [9]. In the case of silicon, the change in the aspect ratios of the photoinduced structures were typically saturated during the delay time of 10 ps between the orthogonally polarized femtosecond double-pulses. Therefore, the time delay between double-pulses was typically fixed to be $1 \mathrm{ps}$ in order to compare to the previous work. Here two thresholds for the internal structural change without surface damage $\left(E^{\text {in }}\right)$ and the surface ablation $\left(E^{\text {sur }}\right)$ should be considered. Furthermore, since the refractive index of GaAs and $\mathrm{GaP}$ is as high as 3.4 and 3.1 respectively at $1.24 \mu \mathrm{m}$, the spherical aberration, leading to the decrease of laser energy density at the focus, should be also compensated in the case of the internal threshold. Fig. 2 shows the threshold energies of $E^{\text {in }}$ and $E^{\text {sur }}$ which are determined from the experiments a function of the focus position from the sample surface. Comparing the threshold to modify the inner part of $\mathrm{GaP}$ and GaAs, the narrow laser energy window was obtained in the case of GaAs. We have also inspected the modified regions embedded in GaAs using infrared microscope and scanning electron microscope (Fig. 3). The photoinduced structure was extended along with the polarization direction (Fig. 3a, $3 b, 3 c)$. Such phenomenon has been also observed in the photoinduced structural changes in $\mathrm{GaP}$ and c-Si. In addition, in the case of this laser condition, the length of the photoinduced structure was about $40 \mu \mathrm{m}$ along the laser propagation (Fig. 3e). We suggested that the excited electron plasma oscillate in the direction parallel to the laser polarization, leading to the structural changes along with the laser polarization direction. Unfortunately, in the case of GaAs, no apparent periodic nanostructures were observed (Fig. 3d, 3f). This phenomenon is similar to the other direct bandgap semiconductors such as $\mathrm{GaN}$ and $\mathrm{ZnO}$.

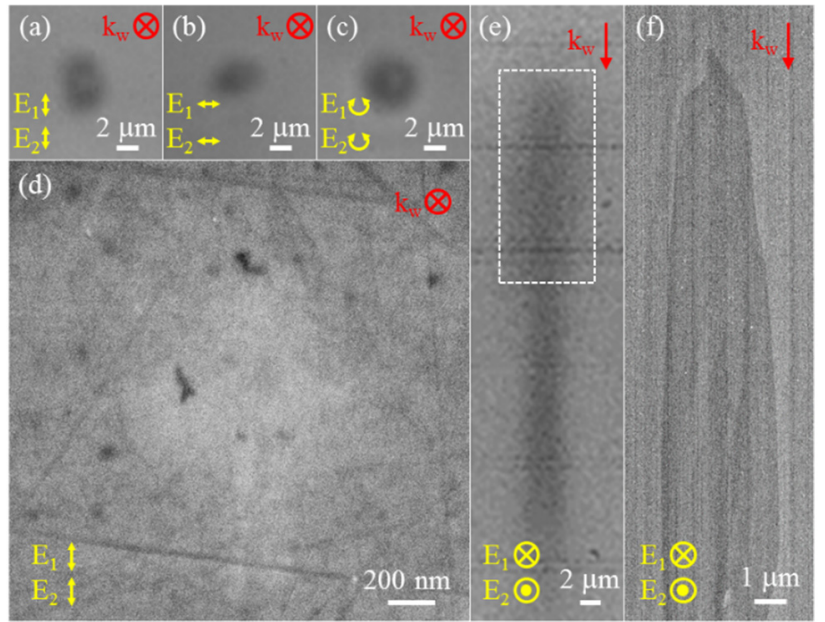

Fig. 3 (a, b, c) IR transmission image of the modified region inside GaAs by IR femtosecond double-pulses combining different polarization directions. (d) SEI on the polished sample surface to the depth of focal position of the femtosecond double-pulse with a total pulse energy of $40 \mu \mathrm{J} . \mathrm{E}_{1}$ and $\mathrm{E}_{2}$ indicate the polarization directions of the first and the second arriving pulse. $\mathrm{k}_{\mathrm{w}}$ shows the laser propagation direction. (e) Cross-sectional IR transmission image of the modified region inside GaAs by IR femtosecond double-pulses. (f) $\mathrm{BEI}$ on the cross-sectionally polished surface corresponding to the dotted area in (e).

It is well known that the secondary electron image (SEI) reveals the surface morphology of a sample, while the 
backscattering electron image (BEI) is sensitive to the atomic weight of the elements or the density of material constituting the observation surface. The brighter contrast region, slightly visible in SEI (Fig. 3d), could be explained by a weak surface relief created in the polishing process. On the other hand, the darker contrast region of the BEI, corresponding to the photoinduced structure, could be interpreted in terms of the defect formation in crystal structure (Fig. 3f). To reveal this suggestion, we have also measured element distribution in the modified region by using Auger electron spectroscopy (AES). Remarkable difference between nonirradiation and irradiation part was, however, not observed. More detailed characterization will be required. It is known that the characteristic Raman peak shift implies an information about local strain in materials. In the case of GaAs crystal, a wurtzite phase enclosed in a zinc-blende structure should be under compressively strained [13]. In Raman spectroscopy, the compressive strains are characterized by a shift toward higher wave numbers. To reveal the local structural changes in GaAs, we analyzed the cleaved (110) surface of the modified region using a confocal Raman spectroscopy (Fig. 4). From the peak shift of the TO phonon peak, we found that the photoinduced structure is compressed. A more detailed study on the Raman spectrum of the induced structure in GaAs is under way.

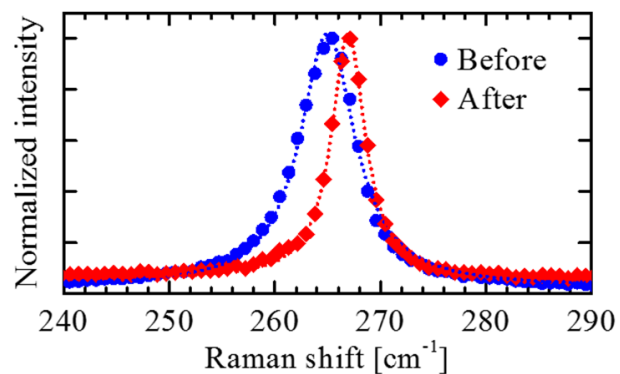

the IR

Fig. 4

femtosecond double-pulses with a total energy of $40 \mu \mathrm{J}$. Raman spectrum of an unirradiated region was also shown. Raman spectra taken using $532 \mathrm{~nm}$ laser excitation. The dotted lines are the Lorentzian fitting curves.

\subsection{Photoinduced structural change in GaP}

It is known that the form birefringence and the negative index change created by the femtosecond laser direct writing in silica glass can be easily characterized by the polarized microscope [14]. The direction of the slow axis of birefringence induced in silica glass is always perpendicular to the writing light polarization [15]. The characterization of irradiated zones induced by the double-pulses with a polarization microscope revealed that nanograting orientation is only defined by the last pulse train, meaning that birefringence is rewritable [10]. We have also characterized the azimuth angle of birefringence of the photoinduced structure in GaP crystal (Fig. 5). Unexpectedly, the photoinduced structures in $\mathrm{GaP}$ indicated form birefringence with the slow axis orientation which is parallel to the laser polarization direction. Furthermore, nanograting in $\mathrm{GaP}$ was aligned parallel to the first arriving pulse polarization without rewriting by the last pulse train. This phenomenon in $\mathrm{GaP}$ is quite different from the case of the silica glass. To reveal the formation of the polarization-dependent nanostructure in crystal, we have also observed the polished sample surface to the depth of the photoinduced structure (Fig. 6).
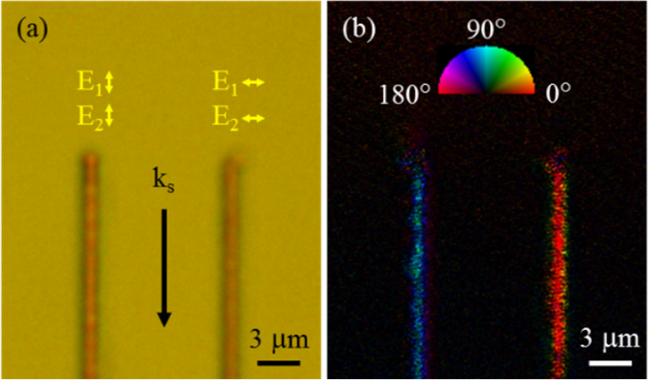

Fig. 5 (a) Transmission and (b) polarization optical microscope images of the laser traces in GaP crystal by the femtosecond doublepulses with the different polarization directions. The total pulse energy was kept at $1 \mu \mathrm{J} . \mathrm{k}_{\mathrm{s}}$ is the laser-scanning direction. Pseudo color in (b) indicates direction of the slow axis, see polar legend. The laser parameters were as follows: $1.24 \mu \mathrm{m}, 110 \mathrm{fs}, 1 \mathrm{kHz}, 5$ $\mu \mathrm{m} / \mathrm{s}, 0.85 \mathrm{NA}$.

A periodic nanostructure with a width of about $210 \mathrm{~nm}$ was formed inside GaP crystal. These results apparently indicate that the periodic nanostructures were self-assembled corresponding to the laser polarization direction. Such relation between the alignment direction of the periodic nanostructure and the laser polarization direction is similar to that of c-Si. In contrast, the principal difference in the periodic nanostructure between $\mathrm{GaP}$ and silica glass is the alignment direction of the periodic nanostructure. Besides, we have also confirmed that the periodic nanostructure was aligned parallel to the polarization direction of the first arriving pulses $\left(E_{1}\right)$, despite of the polarization direction of the secondly arriving pulses $\left(E_{2}\right)$ by using the double-pulse with orthogonally polarization directions (not shown here).
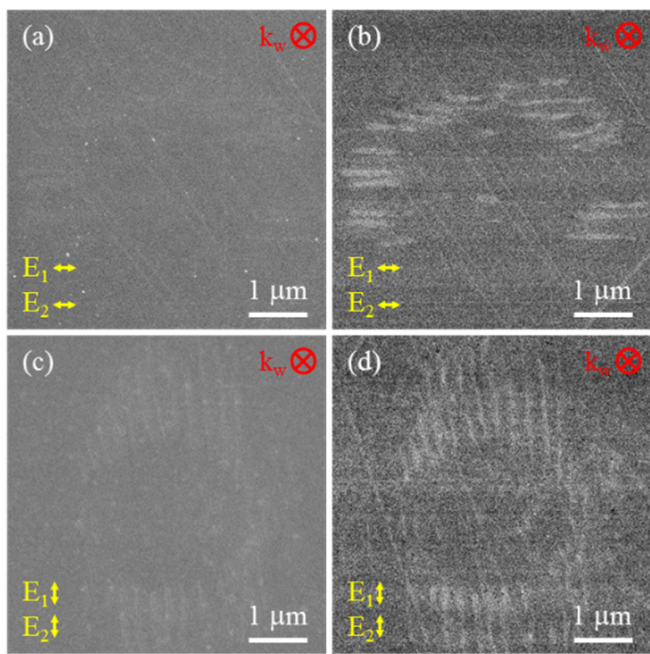

Fig. 6 SEIs $(a, c)$ and BEIs $(b, d)$ of the sample surfaces polished close to the depth of focal spot in GaP crystal. $E_{1}$ and $E_{2}$ indicate the polarization directions of the first and the second arriving pulse. $\mathrm{k}_{\mathrm{w}}$ shows the laser propagation direction. The laser parameters were as follows: $1.24 \mu \mathrm{m}, 110 \mathrm{fs}, 1 \mathrm{kHz}, 10 \mu \mathrm{J}(=5 \mu \mathrm{J} / 5 \mu \mathrm{J}), 0.85$ NA.

It should be noted that the periodic nanostructures inside $\mathrm{GaP}$ were formed around the focal spot location compared to the homogeneous formation in focal volume of silica glass. This is presumably because the electron plasma density generated by the first pulse in the central region is high enough 
to reflect the second pulse. Indeed, since the avalanche ionization is dominant process in the case of semiconductor [9], we assumed that the electron density excited in semiconductor is greater by several orders of magnitude than that of silica glass after several picosecond time delay. By adequately tuning the energy ratio of the double pulse and the time delay, we have also confirmed that the periodic nanostructures can be homogeneously formed at the central part of the focal area as in the silica glass. Since it is known that the plasma density is strongly inhomogeneous both in the propagation direction and the radial direction [16], further investigation including delocalization of femtosecond radiation [17] should be conducted. Another possibility of the nanostructure formed around the focal spot is that since the laser beam at the central region of the focus is entered from the normal direction, the interference with the scattered light is unfavorable compared to around the focus.
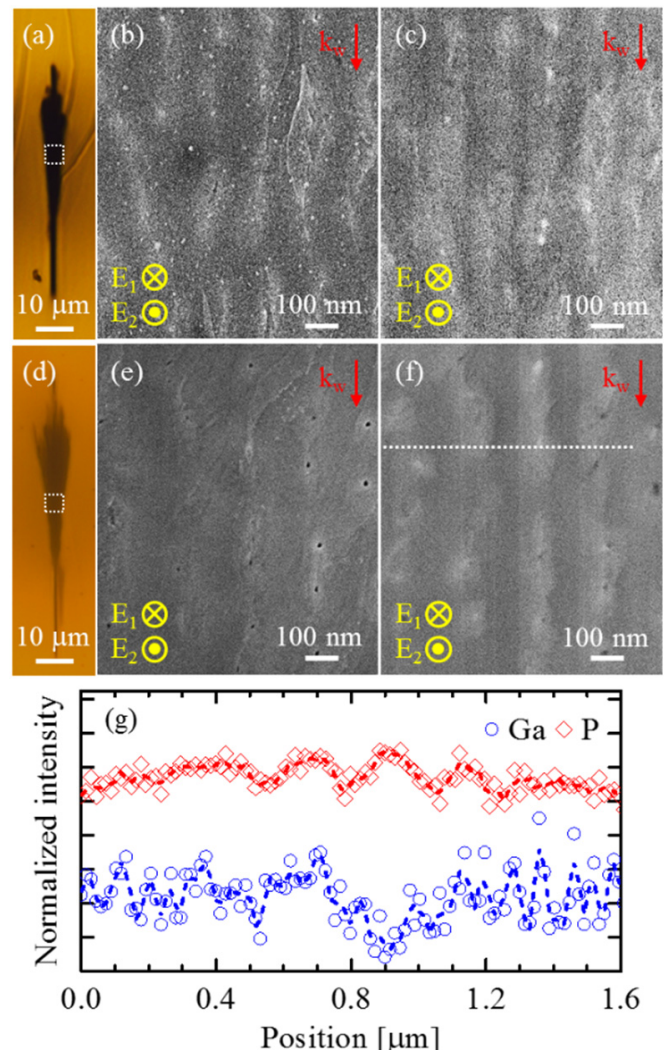

dified

Fig. 7

region induced by the femtosecond double-pulses with a wavelength of $800 \mathrm{~nm}$ (a) and $1240 \mathrm{~nm}$ (b). SEIs (b, e) and BEIs (c, f) on the cleaved surfaces of laser traces written by the femtosecond double-pulses with a different wavelength, corresponding to the dotted area in (a) and (d), respectively. (g) Line profiles of Auger electron spectra (AES) of Ga and $\mathrm{P}$ at the dotted line in (f). The laser parameters were as follows: $110 \mathrm{fs}, 1 \mathrm{kHz}, 10 \mu \mathrm{J}(5 \mu \mathrm{J} / 5 \mu \mathrm{J})$, $5 \mu \mathrm{m} / \mathrm{s}, 0.85 \mathrm{NA}$.

We have also carried out the cross-sectional observation of the photoinduced structures in GaP (Fig. 7). The pitch of the periodic nanostructures created by femtosecond doublepulse with a wavelength of $800 \mathrm{~nm}$ and $1240 \mathrm{~nm}$ were estimated to be about $130 \mathrm{~nm}$ and $210 \mathrm{~nm}$, respectively. The SEIs of the polished sample surface indicates that the morphology could change. Indeed, nanovoids with a size of several tens of nanometers were observed in the brighter region in the SEIs (Fig. 7b, 7e). Furthermore, the BEIs (Fig. 7c, 7f) suggest that the structures around the nanovoids are higher density. The Auger signal of $\mathrm{P}$ in the regions corresponding to bright domains in the BEIs is higher than that of other regions, suggesting high $\mathrm{P}$ concentration in these domains (Fig. 7g). On the other hand, the intensity of the Ga signal is almost same in the whole region. These results indicate that the periodic structures observed in the BEIs, especially surrounding the nanovoids, consist of periodically distributed $\mathrm{P}$ rich regions. We have speculated that this oscillation of $\mathrm{P}$ concentration could be related to the density modulation. Based on the inhomogeneous nanoplasma model [18], the interval of periodic nanostructures inside materials is defined as:

$$
\Lambda=\lambda / 2 \mathrm{n}
$$

where $\lambda$ is the excited laser wavelength, $\mathrm{n}$ is the refractive index of material. From this equation, the intervals for $\lambda=$ $800 \mathrm{~nm}$ and $1240 \mathrm{~nm}$ are evaluated to be $130 \mathrm{~nm}$ and 200 $\mathrm{nm}$, respectively. These results are well agreement with the experimental results.

We have also measured the THz-TDS of GaP crystal before and after the femtosecond double-pulse irradiation (Fig. 8 ). Based on the appropriate Drude model, electrical conductivities were calculated by fitting with the complex refractive index of the sample in $\mathrm{THz}$ region. As the results, the electrical conductivity of laser-treated sample increased about $20 \%$ compared to that of untreated sample. This intriguing phenomenon is probably explained by the formation of strained nanostructure in crystal. The distortion of crystal structures caused by the residual stress after the laser irradiation, leads to the suppression of the lowering of electron mobility by interband and intervalley scattering [19]. Finally, the spatially localized electrical conductivities in $\mathrm{GaP}$ were improved by the laser irradiation.

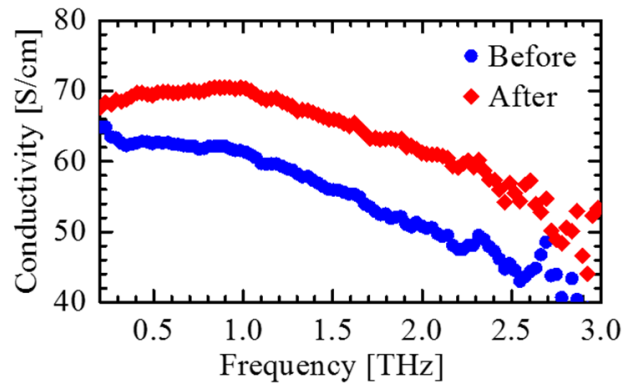

before

Fig. 8

and after the irradiation of the femtosecond double-pulses with a time delay of $1 \mathrm{ps}$. The total pulse energy was $10 \mu \mathrm{J}$. The laser parameters were as follows: $1.24 \mu \mathrm{m}, 110 \mathrm{fs}, 1 \mathrm{kHz}, 5 \mu \mathrm{m} / \mathrm{s}, 0.85 \mathrm{NA}$.

\subsection{Mechanisms of nanostructuring inside indirect bandgap semiconductors}

In our experiments, the periodic nanostructures could not be formed in the direct bandgap semiconductors such as $\mathrm{ZnO}, \mathrm{GaN}, \mathrm{GaAs}$. On the other hand, in the case of indirect bandgap semiconductors of c-Si and GaP, such nanostructures can be created by the femtosecond double pulse irradiation. Other group has also observed that the periodic nanostructure inside $\mathrm{SiC}$ (indirect, $\mathrm{E}_{\mathrm{g}}=3.26 \mathrm{eV}$ ) can be formed [16]. Their results are consistent with our interpretation that the periodic nanostructures can be formed inside a material only if it is an indirect bandgap semiconductor. These phenomena could be interpreted in terms of the difference in the electron transition between indirect and direct 
bandgap semiconductor. When the first arrival femtosecondpulse is focused inside a semiconductor with an indirect bandgap, the free carrier multiplication in focal volume occurs via the two-photon ionization and the avalanche process. The longer relaxation time of the excited carrier in an indirect bandgap promotes the interaction with the time-delayed second pulse, because the radiative recombination in an indirect bandgap semiconductor requires a certain correlation of the positions of not only electrons and holes but also phonons. Finally the subsequent structural modifications inside an indirect bandgap semiconductor are induced. On the other hand, in the case of a direct band gap semiconductor, the carrier (lifetime is sub-picosecond at room temperature) is relaxed before the arrival of time-delayed second pulse. As a result no apparent structural change could be observed. Besides, due to the short plasma lifetime of about $150 \mathrm{fs}$ in silica glass [20], the following formation mechanisms of nanogratings are proposed: the non-radiative relaxation of excitons via relative long lifetime transition from selftrapped excitons (STE) to point-defects (their lifetime is several hundred picoseconds at room temperature) [21]. Especially, a common feature of periodic nanostructure formation between indirect bandgap semiconductor and silica glass is the existence of a transition with a long relaxation time. Although further investigations are required for understanding the mechanisms of self-assembled nanostructure inside an indirect bandgap semiconductor, the formation of such periodic nanostructure is reminiscent of the mechanical vibration of the structure by the stimulated Brillouin scattering involving photon-phonon coupling via electrostriction force [22].

\section{Conclusion}

We experimentally demonstrated that the polarizationdependent periodic nanostructures are successfully induced inside indirect bandgap semiconductors by the infrared femtosecond double-pulse. Especially, nanograting structures inside $\mathrm{GaP}$ are mainly derived from the periodic density variation according to the periodic modulation of $\mathrm{P}$ concentration. Such nanostructures also exhibit higher electrical conductivities. Although more discussions and experiments are required to understand the mechanism, we suppose that the relaxation dynamics of the excited electron is related to the interaction between an electron and a phonon.

\section{Acknowledgments}

This work was partially supported by JSPS KAKENHI Grant Number 26630129, The Thermal \& Electric Energy Technology Foundation, Tokuyama Science Foundation, Cross-Ministerial Strategic Innovation Promotion (SIP) Program and Industry-Academia Collaborative R\&D Programs (Super ClusterProgram).

\section{References}

[1] K. Miura, J. Qiu, H. Inouye, T. Mitsuyu, and K. Hirao: Appl. Phys. Lett., 71, (1997) 3329.

[2] Barada K. Nayak, Vikram V. lyengar, Mool, and C. Gupta: Prog. Photovolt., 19, (2011) 631.

[3] S. Hohm, A. Rosenfeld, J. Kruger, J. Bonse: Appl. Surf. Sci., 278, (2013) 7.

[4] J. Bonse, S. Baudach, J. Kruger, W. Kautek, and M. Lenzner: Appl. Phys. A, 74, (2002) 19.
[5] A. Borowiec and H. K. Haugen: Appl. Phys. Lett., 82, (2003) 4462.

[6] E. M. Hsu, T. H. R. Crawford, C. Maunders, G. A. Botton, and H. K. Haugen: Appl. Phys. Lett., 92, (2008) 221112.

[7] D. Dufft, A. Rosenfeld, S. K. Das, R. Grunwald, and J. Bonse: J. Appl. Phys., 105, (2009) 034908.

[8] J. Bonse, A. Rosenfeld, and J. Kruger: J. Appl. Phys., 106, (2009) 104910.

[9] M. Mori, Y. Shimotsuma, T. Sei, M. Sakakura, K. Miura, and H. Udono: Phys. Status Solidi A, 212, (2015) 715.

[10] Y. Shimotsuma, M. Sakakura, P. G. Kazansky, M. Beresna, J. Qiu, K. Miura, and K. Hirao: Adv. Mater. 22, (2010) 4039.

[11]K. Sugioka, M. Iida, H. Takai, K. Micorikawa: Opt. Lett., 36, (2011) 2734.

[12] Y. Ishikawa, Y. Shimotsuma, A. Kaneta, M. Sakakura, M. Nishi, K. Miura, K. Hirao, and Y. Kawakami: Proc. SPIE, 8243, (2012) 82430N.

[13] I. Zardo, S. Conesa-Boj, F. Peiro, J. R. Morante, J. Arbiol, E. Uccelli, G. Abstreiter, and A. Fontcuberta i Morral: Phys. Rev. B, 80, (2009) 245324.

[14]E. Bricchi, B. G. Klappauf, and P. G. Kazansky: Opt. Lett., 29, (2004) 119.

[15] Y. Shimotsuma, P. G. Kazansky, J. Qiu, and K. Hirao: Phys. Rev. Lett., 91, (2003) 247405.

[16] T. Okada, T. Tomita, S. Matsuo, S. Hashimoto, Y. Ishida, S. Kiyama, and T. Takahashi: J. Appl. Phys., 106, (2009) 054307.

[17] V. V. Kononenko, V. V. Konov and E. M. Dianov: Opt. Lett., 37, (2012) 3369.

[18]P. P. Rajeev, M. Gertsvolf, C. Hnatovsky, E. Simova, R. S. Taylor, P. B. Corkum, D. M. Rayner, and V. R. Bhardwaj: J. Phys. B: At. Mol. Opt. Phys., 40, (2007) S273.

[19] Y. Sun, S. E. Thompson, and T. Nishida, J. Appl. Phys., 101, (2007) 104503.

[20] M. Lancry, N. Groothoff, B. Poumellec, S. Guizard, N. Fedorov, and J. Canning: Phys. Rev. B, 84, (2011) 245103.

[21]D. Wortmann, M. Ramme, and J. Gottmann: Opt. Express, 15, (2007) 10149 .

[22] R. Boyd: "Nonlinear Optics", (Academic Press, San Diego, 2003) p.416.

(Received: May 25, 2015, Accepted: January 23, 2016) 